\title{
Evaluation of solid pseudopapillary tumor of the pancreas: one tertiary center experience.
}

\author{
Yusuf Kayar $^{1 *}$, Nurcan Unver ${ }^{2}$, Ganime Coban², Zuhal Gucin², Iskender Ekinci', Sebnem Emegil ${ }^{1}$, \\ Nuket Bayram Kayar ${ }^{3}$, Birol Baysal ${ }^{1}$, Mehmet Sait Bugdaci ${ }^{1}$, Hakan Senturk ${ }^{1}$ \\ ${ }^{1}$ Faculty of Medicine, Department of Internal Medicine, Division of Gastroenterology, Bezmialem Vakif University, \\ Istanbul, Turkey \\ ${ }^{2}$ Faculty of Medicine, Department of Pathology, Division of Gastroenterology, Bezmialem Vakif University, Istanbul, \\ Turkey \\ ${ }^{3}$ Department of Family Medicine, Bagcilar Education and Training Hospital, Istanbul, Turkey
}

\begin{abstract}
Solid Pseudo-Papillary Neoplasia (SPN) is the rare tumors of the pancreas. The aim of the current study is documentation of the data of the patients. The data of patients with pancreatic neoplasm were presented to Gastroenterology Department between 2010 and 2014 were evaluated retrospectively. A database of the characteristics of these patients was developed, including demographic, laboratory, radiological, surgical and pathological features and the immuno-histochemical staining of tumor specimens. Analysis of 323 patients diagnosed with pancreas malignancies in our hospital between 2010 and 2014 demonstrated that 5 patients $(1.5 \%)$ were diagnosed with SPN. Of these five patients, four were females and one was male. Their average age was 27 (range: 16-50). CA 19-9 levels were normal in four patients while slightly increase in one $(60 \mathrm{U} / \mathrm{mL}(0-33))$ and there were the other tumor markers under a normal range $5-10.3 \mathrm{~cm}$ diameter in size, mean: $7.6 \mathrm{~cm}$. Immunohistochemical analysis demonstrated that CD10, CD56, Ki-67 and $\beta$-catenine was positive for all patients while other markers were variable. Progesteron and estrogen receptors were positive in histo-pathologic specimens of four patients. SPN is a rare tumor that develops principally in young women and has a good prognosis. Immunohistochemical staining is important in the differential diagnosis of SPN and estrogen positivity may have a role in pathogenesis of a limited patients.
\end{abstract}

Keywords: Solid pseudopapillary neoplasia (SPN), Pancreatic mass, Cystic pancreatic tumor.

Accepted on January 09, 2018

\section{Introduction}

Solid Pseudopapillary Neoplasia (SPN) of the pancreas is a neoplasm with a low malignant potential, usually affecting young women in the second or third decade of life [1]. First reported by Frantz in 1959, it is an uncommon but distinct pancreatic neoplasm, constitutes only about $5 \%$ of cystic pancreatic tumors and about 1 to $2 \%$ of exocrine pancreatic neoplasms [2-5]. There is an increase of incidence of the disease with widely availability of imaging systems and better understanding of its pathology.

Data in regard with the disease were scarce. We aimed in the current study is to analyze the data of the patients diagnosed with SPN, to evaluate them in connection with other cases previously reported in the literature and to discuss the management of SPN as a rare entity.

\section{Methods}

Five patients who were diagnosed and treated for SPN at the Gastroenterology Department of Bezmialem Vakif University between 2010 and 2014 were evaluated retrospectively. A database of the characteristics of these patients was developed, including age, gender, symptoms, duration of symptoms, tumor location (data were from radiological investigations or surgical record) and size (data were from radiological investigations or surgical record and finally confirmed by pathology), metastasis or invasion of adjacent tissues (data were from radiological investigations or surgical exploration, and finally was confirmed by pathology), histopathological and immunohistochemical features, treatment (data were from the record of therapy, including the types of surgery) and followup. In addition, comorbidities, hepatic function test, amylase, lipase values, tumor markers ( $\alpha$-Fetoprotein (AFP), CarcinoEmbryonic Antigen (CEA), Carbohydrate Antigen (CA) 19-9, CA-125) was also documented. Imaging results of all patients 
(Ultrasonography (USG), Computed tomography (CT), Magnetic Resonance (MR)) and preoperative Endoscopic Ultrasonography (EUS)-Fine Needle Aspiration (FNA) were evaluated.

\section{Results}

The retrospective analysis of 323 patients diagnosed with pancreas malignancies in our hospital between 2010 and 2014 demonstrated that 5 patients $(1.5 \%)$ were diagnosed with SPN. Of these five patients, four were females and one was male. Their average age was $27 \pm 14 / y$, (range: 16-50). All patients had abdominal pain and discomfort while three patients had only nausea/vomiting and one had palpable abdominal mass. One patient had varicosele and one had hypertension as concomitant conditions while others had none. All patients' hepatic function tests and amylase/lipase measurements were normal.

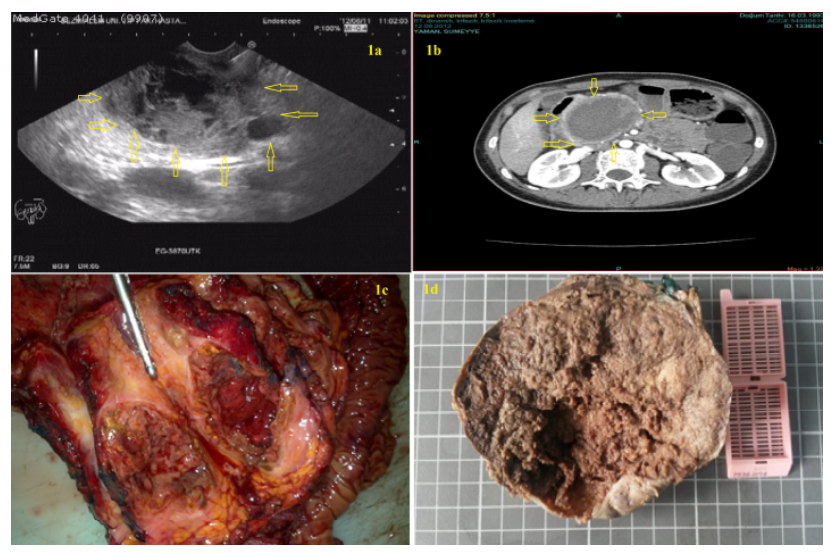

Figure 1. (a) $7 \mathrm{~cm}$ diameter lesion in the pancreatic head consistent with necrotic and cystic areas; (b) $7 \times 6 \times 5 \mathrm{~cm}$ diameter lesion in the pancreatic head-uncinate area. The complex cystic mass has a thick irregular rim and contains solid components; (c) Tumor which welldefined cystic tumors and hemorrhagic discharges in the pancreatic head; (d) Pancreatic tumors well-defined structures consistent with pseudopapillary structures associated bleeding and necrosis.

Likewise, all patients CEA, CA 125, AFP values were normal. CA 19-9 levels were normal in four patients while slightly increased in one $(60 \mathrm{U} / \mathrm{mL}(0-33))$. Tumors of three patients were localized at the pancreas tail and at the head in two patients (Figure 1a). Extrahepatic involvement was not observed for any of the patients. Tumor diameters ranged between $5 \mathrm{~cm}$ and $10.3 \mathrm{~cm}$, with an average diameter of $7.6 \mathrm{~cm}$ (Table 1).

Abdominal CT and MR demonstrated typical features of SPN for only two patients (Figure 1b). Four patients underwent EUS-FNA while one patient had no EUS. Two of the four patients who had undergone EUS-FNA were diagnosed with
SPN, one patient was misdiagnosed (ependymoma) and one patient did not receive any diagnosis. Of the patients who had undergone surgical intervention, one received distal pancreatectomy, two received Whipple surgery and two received distal pancreatectomy and splenectomy (Table 1).

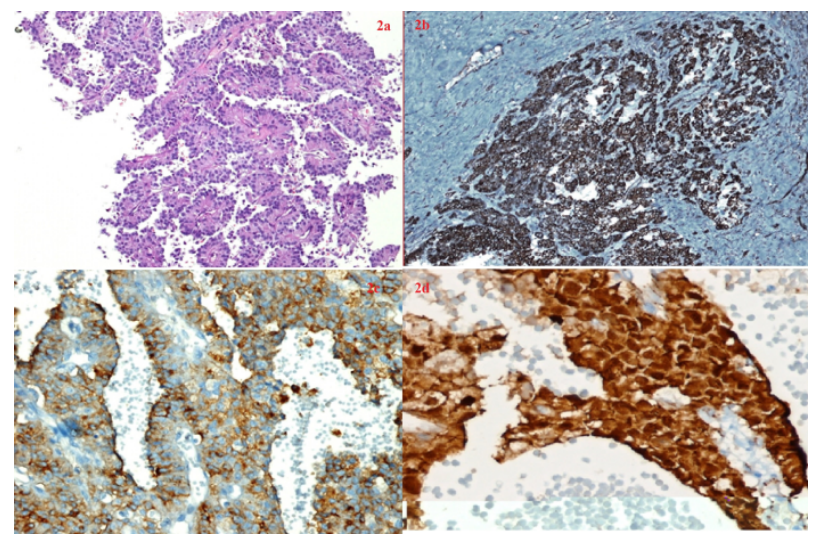

Figure 2. (a) General view with hematoxylin and eosin, pseudopapillary structures (H and $E \times 40)$; (b) Vimentin $\times 40$; $(c)$ : Cytoplasmic staining with CD10; (d) Nuclear expression with Beta catenin $\times 400$.

Macroscopically, there was diffuse hemorrhage between solid and cystic areas in all patients. At histopathological examination, tumor mass was separated from pancreas with a fibrous capsula. Pseudopapillary, cystic and solid growth patterns were seen in the tumor mass.

Tumor cells had an ovally-shaped, small and centrally localized nucleus and large eosinophilic cytoplasm. Tumors consisted of pseudopapillary structures made of cells aligned around fine vessels, solid areas, hemorrhagic areas and cystic areas of different size (Figure 1c, 1d and 2a).

Mitosis was not observed in two patients while three had minimal mitosis $(1 / 10)$. Two patients had no necrosis while necrotic areas were observed within the tumor for three patients. Extra-pancreatic or lymph node invasion was not observed with pathologic examination in any of the patients (Table 2).

Immunohistochemical analysis demonstrated that vimentin (Figure 2b), CD10 (Figure 2c), CD56, Ki-67 and B-catenin (Figure 2d) was positive for all patients while other markers were variable.

Progesteron and estrogen receptors were positive in histopathologic specimens of four patients (Table 3). Although the average follow-up duration was 8.8 months (range: 2-21 months), all patients are being followed up without complications.

Table 1. Demographic data of 5 SPN cases.

\begin{tabular}{llllll}
\hline Patients & Patient 1 & Patient 2 & Patient 3 & Patient 5 & Patient 4 \\
\hline Age $(\mathrm{y})$ & 31 & 18 & 50 & 16 \\
\hline
\end{tabular}


Evaluation of solid pseudopapillary tumor of the pancreas: one tertiary center experience

\begin{tabular}{|c|c|c|c|c|c|}
\hline Gender & Male & Female & Female & Female & Female \\
\hline Symptom & Abdominal pain, Discomfort & $\begin{array}{lr}\text { Abdominal } & \text { pain, } \\
\text { Discomfort, } & \text { Nausea, } \\
\text { Vomiting } & \end{array}$ & $\begin{array}{l}\text { Abdominal pain, Discomfort, } \\
\text { Nausea, Vomiting }\end{array}$ & $\begin{array}{l}\text { Abdominal pain, } \\
\text { Discomfort, Nausea, } \\
\text { Abdominal mass }\end{array}$ & $\begin{array}{l}\text { Abdominal pain, } \\
\text { Discomfort }\end{array}$ \\
\hline Initiation of Symptoms & 2 months & 5 months & 16 months & 4 months & 1 month \\
\hline Comorbidities & Varicosele & None & Hypertension & None & None \\
\hline LFT elevated & Normal & Normal & Normal & Normal & Normal \\
\hline Amylase/Lipase & Normal & Normal & Normal & Normal & Normal \\
\hline CEA/CA19-9/AFP & Normal & Normal & Normal & $\begin{array}{l}\text { Normal/60 U/mL(0-33)/ } \\
\text { Normal }\end{array}$ & Normal \\
\hline Localization & Tail & Head & Tail & Tail & Head \\
\hline Dimension & $65 \times 60 \mathrm{~mm}$ & $50 \times 30 \mathrm{~mm}$ & $93 \times 75 \mathrm{~mm}$ & $103 \times 82 \mathrm{~mm}$ & $70 \times 60 \mathrm{~mm}$ \\
\hline Metastasis & None & None & None & None & None \\
\hline Lymph node involvement & None & None & None & None & None \\
\hline Surgery type & Distal Pancreatec-tomy & Whipple's surgery & Distal Pancreatec-tomy & Distal Pancreatec-tomy & $\begin{array}{l}\text { Whipple's } \\
\text { surgery }\end{array}$ \\
\hline Follow-up & 13 months, healthy & 6 months, healthy & 2 months, healthy & 2 months, healthy & $\begin{array}{l}21 \text { months, } \\
\text { healthy }\end{array}$ \\
\hline
\end{tabular}

SPN: Solid pseudo-papillary neoplasia; LFT: Liver function tests; mm: Millimeter.

Table 2. Histopathologic evaluation of SPN patients.

\begin{tabular}{|c|c|c|c|c|c|}
\hline & Patient 1 & Patient 2 & Patient 3 & Patient 4 & Patient 5 \\
\hline Solid/Cystic & Solid/Cystic & Solid/Cystic & Solid/Cystic & Solid/Cystic & Solid/Cystic \\
\hline Necrosis & Minimal & None & Yes & Yes & None \\
\hline Mitosis & 43374 & None & 43374 & 43374 & None \\
\hline Invasion & None & None & None & None & None \\
\hline Lymph node involvement & None & None & None & None & None \\
\hline
\end{tabular}

Table 3. Immunohistochemical findings of the pathologic specimens of SPN's of 5 cases.

\begin{tabular}{|c|c|c|c|c|c|}
\hline & Patient 1 & Patient 2 & Patient 3 & Patient 4 & Patient 5 \\
\hline Cytokeratin & $(-)$ & $(+)$ & $(+)$ & $(+)$ & $(+)$ \\
\hline CEA & $(-)$ & $(-)$ & $(-)$ & $(-)$ & $(-)$ \\
\hline Vimentin & $(+)$ & $(+)$ & $(+)$ & $(+)$ & $(+)$ \\
\hline Chromogranin & $(-)$ & $(-)$ & $(-)$ & $(-)$ & $(-)$ \\
\hline NSE & $(+)$ & $(-)$ & $(+)$ weak focally & $(-)$ & $(+)$ \\
\hline Synaptophysin & $(+)$ & $(+)$ focally & $(+)$ focally & $(-)$ & $(-)$ \\
\hline CD10 & $(+)$ & $(+)$ focally & $(+)$ & $(+)$ & $(+)$ \\
\hline CD56 & $(+)$ & $(+)$ & $(+)$ & $(+)$ & $(+)$ \\
\hline P53 & $(+)$ isolate & $(+)$ rarely & $(+)$ isolate & $(+)$ & $(+)$ mild \\
\hline Ki-67 index & (+) $2 \%$ & (+) $3-5 \%$ & (+) $5-10 \%$ & $(+) \% 10$ & $(+) \% 2$ \\
\hline Progesteron & $(+)$ focally & $(+)$ focally & $(+)$ focally & $(+)$ & $(-)$ \\
\hline
\end{tabular}




\begin{tabular}{|c|c|c|c|c|c|}
\hline Estrogen & $(+)$ weak isolate & $(+)$ rarely & $(-)$ & $(+)$ & $(+)$ rarely \\
\hline B-Catenin & $(+)$ & $(+)$ & $(+)$ & $(+)$ & $(+)$ \\
\hline EMA & $(-)$ & $(-)$ & $(-)$ & $(-)$ & $(-)$ \\
\hline S100 & $(-)$ & $(+)$ focally & $(-)$ & $(-)$ & $(+)$ \\
\hline
\end{tabular}

Positive in isolate cell: $1 \% \downarrow$; Rare: 1-10\%; Mild: 10-50\%; (+): Common: 50\%个.

\section{Discussion}

SPN of the pancreas is a neoplasm with a low malignant potential, usually affecting young women in the second or third decade of life [1]. SPN is very rare; in fact, it only constitutes about $5 \%$ of cystic pancreatic tumors and about 1 to $2 \%$ of exocrine pancreatic neoplasms [4,5]. The five patients in our study age between 16 and $50 \mathrm{y}$, with a mean age of $27 \mathrm{y}$, consistent with the literature $[4,6,7]$.

The clinical presentation of SPN is usually unspecific. Most patients have unclear clinical features including abdominal pain or discomfort, poor appetite and nausea, which are related to tumor compression on adjacent organs. Pain is the most pronounced complaint in these patients [1]. In our study, all patients had abdominal pain and abdominal discomfort while two patients had additional complaints of nausea/vomiting and one had palpable mass in the abdomen. Usually there is no evidence of pancreatic insufficiency, abnormal liver function tests, cholestasis, elevated pancreatic enzymes or an endocrine syndrome. Tumor markers are also generally unremarkable [4-8]. Hepatic function tests and pancreatic enzymes were normal in all patients consistent with previous reports. One patient had moderately high levels of CA19-9, while tumor markers were of normal levels in others.

Today's imaging techniques are helpful for differentiation of SPN from other cystic neoplasms such as serous cystadenomas, mucin-producing tumors and islet cell tumors. A well demarcated encapsulated lesion including solid and cystic components and hemorrhagic degenerations are seen by CT [9]. Following contrast material administration, enhancing solid areas are typically noted peripherally, whereas cystic spaces are usually more centrally located [10]. Cystic mass may have peripheral curvilinear calcification and solid mass have necrotic areas in it [11]. As reflecting complex nature of SPN, MRI imaging demonstrates a well-defined lesion with heterogeneous signal intensity on T1 and T2-weighted images. Blood product in the SPNs that caused by internal hemorrhage can be diagnosed by increase in signal attenuation in the T1 weighted and decrease in T2-weighted images [9]. MRI may also help in differentiation between SPNs and islet cell tumors; cystic components have moderately increased signal intensity on T1-weighted images and increased signal intensity on T2weighted images in islet cell tumors. Increased peripheral hypervascularity is seen in the islet cell tumors but not in SPNs [10]. EUS-guided fine-needle aspiration (or percutaneous) may play an important role in distinguishing SPNs from other lesions and preoperative planning [12]. Preoperative diagnosis, however, is still a challenge despite technological advances.
Combined assessment of imaging results and EUS-FNA with consideration to age and gender will facilitate establishing the diagnosis.

The origin of solid pseudopapillary tumors still remains unclear. Absence of communication with the pancreatic duct and mucinous or serous epithelium usually supported by an "ovarian" stroma are the easier differentiation elements of SPNs from cystic neoplasms, such as mucinous or serous cystic neoplasm [13]. SPNs might originate from the genital ridge-related cells that were incorporated into the pancreas during organogenesis. Morales et al. showed fast growth of SPN during pregnancy and they also demonstrated the presence of a progesterone receptor in the tumor tissue [14,15]. The growth rate of the SPN of the pancreas seemed to be enhanced by the concurrence of pregnancy. The predilection for solid-pseudo papillary neoplasm in the young woman suggests a role for sex hormones in the histogenesis or progression of this tumor; however, the anti-estrogen receptor (ER) and anti-progesterone receptor (PR) expression was not consistent in the literature. Using a binding assay, Ladanyi et al. demonstrated the expression of ER $(\mathrm{Kd}=2.82 \pm 0.86 \mathrm{nM})$ in solid-pseudo papillary neoplasm and proposed that this tumor is hormone sensitive (15). However, Carbone et al. reported expression of a type of ER with a $\mathrm{Kd}=15 \mathrm{nM}$ (but not the expression of the high affinity type of $\mathrm{ER}(\mathrm{Kd}=0.1 \mathrm{nM}))$ in solid-pseudo papillary neoplasms [16]. In the same report, the authors demonstrated the lack of ER expression by immunohistochemical staining and suggested that the anti-ER antibodies used in immunohistochemical studies were only specific for high affinity type ER. This assumption may explain the reported lack of ER expression in solidpseudopapillary neoplasm stained with immunohistochemistry in the literature. In our study $80 \%$ of our cases have had estrogen positivity. We suggest that positivity of estrogen may play a role in the pathogenesis of SPN although positivity and the role of estrogen controversial.

A new immunohistochemical feature of SPNs was found recently, a nuclear type of $\beta$-catenin, which were present in more than $90 \%$ of them [17]. It has been shown that, abnormal nuclear labeling of $\beta$-catenin supports the diagnosis [18]. $\beta$ catenin and Wnt signaling pathway play an important role in the tumorigenesis of SPNs [18]. By blocking the degradation of $\beta$-catenin with gene mutations (CTNNB1), the $\beta$-cateninTcf/Lef complex activates the transcription of several oncogenic genes, including c-myc and cyclin D1 in the nucleus [13]. It has been shown that, $\beta$-catenin activation in mice induces large pancreatic tumor resembling human SPN [13]. 
Aggressivity may be decided by presence of extensive necrosis, nuclear atypia and high mitotic rate, immunohistochemistry findings of expression of $\mathrm{Ki}-67$ and sarcomatoid areas in SPNs and about $15 \%$ of the SPNs metastases mostly to the liver, regional lymph nodes, mesentery, omentum and peritoneum as well as adjacent organs $[4,19]$. There was no metastatic lesion in our patients.

Differentiation between the well differentiated neuroendocrine tumors and SPNs may be difficult because of presence of resembling light microscopic features, and variably expressed neuroendocrine markers in SPN [13-20]. While chromogranin A was negative, expressions of other neuroendocrine markers are variously positive such as synaptophysin, neuron-specific enolase, CD10 and CD56 [13-20].

Radical resection is the treatment of choice for SPNs even with metastasis or local recurrence. Distal pancreatectomy combined with or without splenectomy can be performed for pancreatic body and/or tail tumor, and pancreatoduodenectomy for pancreatic head tumor. Martin et al. reported that four patients underwent resection of liver metastasis and primary tumor, and two of them survived for at least 6 and 11 years, respectively [3]. Other treatment modalities for liver metastasis, such as alcohol injection, transarterial chemoembolization, $\gamma$-radiation therapy and even liver transplantation, have been reported [21]. The role of chemotherapy and radiotherapy in treatment of SPN is poorly defined at present, since only few reports are available on them [4]. The prognosis of SPN patients even with local recurrence and metastasis or invasion is good. SPN is limited to the pancreas in over $95 \%$ of its patients and can be radically resected [7]. Its local recurrence rate is less than $10 \%$ and usually occurs within 4 years after surgery [22]. Recurrence, local invasion, and limited metastasis are not contraindications for resection, and some patients with "unresectable" SPN may also have a long survival time [23]. It has been reported that the overall 5-y survival rate of SPN patients is about 95\% [20].

\section{Conclusion}

SPN is a rare tumor that develops principally in young women and has a good prognosis. There is an increase in the rate of diagnosis parallel to technological advances. Surgical intervention should be undertaken as the first choice because it is considerably curative.

\section{References}

1. Guerrache Y, Soyer P, Dohan A, Faraoun SA, Laurent V, Tasu JP. Solid-pseudo papillary tumor of the pancreas: MR imaging findings in 21 patients. Clin Imaging 2014; 38: 475-482.

2. Frantz VK. Tumors of the pancreas. In: Atlas of tumor pathology. Washington, DC: Armed forces Institute of Pathology 1959; 32-33

3. Martin RC, Klimstra DS, Brennan MF, Conlon KC. Solid pseudopapillary tumor of the pancreas: a surgical enigma? Ann Surg Oncol 2002; 9: 35-40
4. Yu PF, Hu ZH, Wang XB, Guo JM, Cheng XD, Zhang YL. Solid pseudopapillary tumor of the pancreas: a review of 553 cases in Chinese literature. World J Gastroenterol 2010; 16: 1209-1214.

5. Klimstra DS, Wenig BM, Heffess CS. Solid pseudopapillary tumor of the pancreas: a typically cystic carcinoma of low malignant potential. Semin Diagn Pathol 2000; 17: 66-80.

6. Kloppel G, Solcia E, Longnecker DS, Capella C, Sobin LH: Histological typing of tumors of the exocrine pancreas. In World Health Organization International Histological Classification of Tumours. Springer 2nd Edition, Berlin, Heidelberg, New York 1996; 8452/1.

7. Lam KY, Lo CY, Fan ST. Pancreatic solid-cystic-papillary tumor: Clinicopathologic features in eight patients from Hong Kong and review of the literature. World J Surg 1999; 23: 1045-1050.

8. Yagcı A, Yakan S, Coskun A, Erkan N, Yıldırım M, Yalcın E. Diagnosis and treatment of solid pseudopapillary tumor of the pancreas: experience of one single institution from Turkey. World J Surg Oncol 2013; 11: 308.

9. Kristin M. Coleman, Michael C. Doherty, Steven AB. Solid-Pseudopapillary Tumor of the Pancreas. Radio Graphics 2003; 23: 1644-1648.

10. Kehagias D, Smyrniotis V, Gouliamos A, Vlahos L. Cystic pancreatic neoplasms: computed tomography and magnetic resonance imaging findings. Int J Pancreatol 2000; 28: 223-230.

11. Kim JJ, Cheon YK, Han HS, Shim CS. Metastatic malignant solid pseudopapillary tumor of the pancreas. Korean J Intern Med 2013; 28: 748-749.

12. Buchino JJ. Fine-needle aspiration of solid and papillary cystic tumor of the pancreas. Pediatr Pathol Lab Med 1996; 16: $235-242$.

13. Zhi-Wei G, Lu S, Yan-Qiu W, Bai-Xuan X. Solid pseudopapillary tumor of the pancreas and concomitant urogenital malformations in a young woman. Diagnos Pathol 2013; 8: 35.

14. Morales A, Ruiz Molina JM, Esteves HO, Robles-Diaz G, Diaz-Sanchez V. Papillary-cystic neoplasm of the pancreas: a sex-steroid dependent tumor. Int J Pancreatol 1998; 24: 219-225.

15. Ladanyi M, Mulay S, Arseneau J, Bettez P. Estrogen and progesterone receptor determination in the papillary cystic neoplasm of the pancreas. With immunohistochemical and ultra-structural observations. Cancer 1987; 60: 1604-1611.

16. Carbone A, Ranelletti FO, Rinelli A, Vecchio FM, Lauriola L, Piantelli M. Type II estrogen receptors in the papillary cystic tumor of the pancreas. Am J Clin Pathol 1989; 92: 572-576.

17. Cavard C, Audebourg A, Letourneur F, Audard V, Beuvon F, Cagnard N. Gene expression profiling provides insights into the pathways involved in solid pseudopapillary neoplasm of the pancreas. J Pathol 2009; 218: 201-209.

18. Shimada K, Nakamoto Y, Isoda H, Maetani Y, Yamashita R, Arizono S. F-18 fluorodeoxyglucose uptake in a solid 
pseudopapillary tumor of the pancreas mimicking malignancy. Clin Nucl Med 2008; 33: 766-768.

19. Washington K. Solid-pseudopapillary tumor of the pancreas: challenges presented by an unusual pancreatic neoplasm. Ann Surg Oncol 2002; 9: 3-4.

20. Papavramidis T, Papavramidis S. Solid pseudopapillary tumors of the pancreas: review of 718 patients reported in English literature. J Am Coll Surg 2005; 200: 965-972.

21. Sun GQ, Chen CQ, Yao JY, Shi HP, He YL, Zhan WH. Diagnosis and treatment of solid pseudopapillary tumor of pancreas: a report of 8 cases with review of domestic literature. Zhonghua Putong Waike Zazhi 2008; 17: 902-907.

22. Sperti C, Berselli M, Pasquali C, Pastorelli D, Pedrazzoli S. Aggressive behaviour of solid-pseudopapillary tumor of the pancreas in adults: a case report and review of the literature. World J Gastroenterol 2008; 14: 960-965.
23. Mao C, Guvendi M, Domenico DR, Kim K, Thomford NR, Howard JM. Papillary cystic and solid tumors of the pancreas: a pancreatic embryonic tumor? Studies of three cases and cumulative review of the world's literature. Surgery 1995; 118: 821-828.

\section{*Correspondence to}

Yusuf Kayar

Faculty of Medicine

Department of Internal Medicine

Division of Gastroenterology

Bezmialem Vakıf University

Istanbul

Turkey 